\title{
Floração e morfologia floral de variedades de melancieira em Juazeiro-BA
}

\section{Flowering and floral morphology of watermelon varieties in Juazeiro-BA}

\author{
Poliana Martins Duarte ${ }^{1}$, Kátia Maria Medeiros de Siqueira ${ }^{2}$, Natália Campos da Silva ${ }^{3}$, Gleydson Brenno Santos Silva ${ }^{4}$, Ítala \\ Laiane Silva Gomes ${ }^{5}$
}

\begin{abstract}
Resumo- A melancieira apresenta comumente flores masculinas e femininas na mesma planta, necessitando assim dos serviços de polinização para a transferência dos grãos de pólen e consequente produção de frutos. A atratividade aos polinizadores está na dependência dos recursos florais oferecidos como recompensa durante as visitas, e como consequência a planta é beneficiada com a polinização. O objetivo desse trabalho foi registrar aspectos relevantes da floração incluindo a razão sexual e a descrição da morfologia floral de variedades de melancieira. As variedades demelancieira utilizadas foram, Congo, Elisa, Crimson super e Charleston super, em cultivo orgânico, com irrigação por gotejamento. Foram avaliados quatro tratamentos e quatro repetições, utilizando o delineamento experimental inteiramente casualizado. Para todas as variedades analisadas, foi registrada a emissão inicial de flores masculinas. Não foi registrada diferença significativa na emissão de flores femininas entre as variedades, porém para as flores masculinas a variedade Congo apresentou um maior número. Foi registrada diferença nas características da morfologia floral entre variedades e tipos florais em relação ao diâmetro e altura da flor. A presença de flores hermafroditas foi registrada com média de 1,45 (Crimson) a 0,15 flores por planta (Congo). A variedade Charleston apresentou a menor média de dias para a emissão da primeira flor feminina em relação a masculina $(4,6 \pm 2,81)$ já a Congo apresentou a maior média $(9,05 \pm 3,28)$. A menor razão sexual foi registrada para a variedade Elisa $(1: 13,32)$ e a maior para a Congo $(1: 24,05)$. Os menores períodos de floração foram registrados para as variedades Crimson e Elisa.
\end{abstract}

Palavras-chave- Citrulus lanatu; biologia floral; razão sexual

Abstract- The watermelon commonly presents male and female flowers on the same plant, thus requiring the pollination services for the transfer of pollen grains and consequent production of fruits. The attractiveness to pollinators is dependent on the floral resources offered as a reward during visits and as a result the plant benefits from pollination. The objective of this study was to record important aspects of flowering including sex ratio and the description of floral morphology of varieties of watermelon. The varieties of watermelon were used, Congo, Elisa, super super Crimson and Charleston in organic farming, drip irrigation. Four treatments and four repetitions were performed using a completely randomized design. For all varieties analyzed, it was registered the initial issue of male flowers. It did not record significant difference in the issue of female flowers between varieties, but the male flowers to the Congo variety presented more. There was recorded differences in the characteristics of morphology flower varieties and types in relation to the height and flower diameter. The presence of hermaphrodite flowers was recorded with an average of 1.45 (Crimson) 0.15 flowers per plant (Congo). The Charleston variety had the lowest average number of days to issue the first female flower compared to males $(4.6 \pm 2.81)$ since the Congo had the highest average $(9.05 \pm 3.28)$. The lowest sex ratio was recorded for the variety Elisa $(1: 13.32)$ and the highest for the Congo (1: 24.05). The smallest flowering periods were recorded for the Crimson and Elisa varieties.

Key words- Citrulus lanatus; floral biology; sex ratio

\footnotetext{
*Autor para correspondência

Recebido para publicação em 01/06/2015; aprovado em 15/09/2015

${ }^{1}$ Graduanda em Engenharia Agronômica, bolsista de iniciação cientifica Fundação de Amparo a Pesquisa do Estado da Bahia (FAPESB). E-mail: eng.poliana_duarte@hotmail.com

${ }^{2}$ Professora Titular da Universidade do Estado da Bahia - Campus III - DTCS - Juazeiro-BA. E-mail: katiauneb@yahoo.com.br

${ }^{3}$ Graduanda em Engenharia Agronômica, bolsista de iniciação cientifica Fundação de Amparo a Pesquisa do Estado da Bahia (FAPESB). E-mail: naty_32campos@hotmail.com

${ }^{4}$ Graduanda em Engenharia Agronômica, bolsista de iniciação cientifica Fundação de Amparo a Pesquisa do Estado da Bahia (FAPESB). E-mail: gleydsonuneb@gmail.com

5. Universidade do Estado da Bahia - Campus III - Departamento de Tecnologia e Ciências Sociais (DTCS)- Juazeiro- BA. E-mail: itala.gomes@ hotmail.com
} 


\section{INTRODUÇÃO}

A melancieira (Citrullus lanatus (Thunb.) Matsum \& Nakai), pertence a família Cucurbitaceae, tendo sua origem na África, sendo amplamente cultivada nas regiões tropicais, semitropicais e áridas de todo o mundo (ALMEIDA, 2003). Seus frutos são uma importante fonte de vitaminas, minerais, carotenóides e proteínas, bem como considerados como uma rica fonte de licopeno natural, um carotenóide de grande importância por causa de sua propriedade antioxidante (DENTON, 2004).

Esta cucurbitácea apresenta flores solitárias, femininas e masculinas, podendo de acordo com o cultivar, apresentar flores hermafroditas em pequeno número. Estas flores são atrativas aos visitantes por fornecer pólen e néctar como recompensa floral. Durante o forrageamento as abelhas visitam ambos os tipos florais, porém as flores masculinas disponibilizam pólen e néctar, tornando-se assim mais atrativas.

No Kenya, estudos realizados com a melancieira indicaram que as abelhas Apis mellifera visitaram em maior número as flores masculinas, porém permaneceram mais tempo nas flores femininas, o que pode ser benéfico para a polinização, uma vez que, quando as abelhas visitam as flores femininas já estão com bastante pólen no corpo, o que garante uma polinização efetiva (NJOROGE, 2004)

Atributos florais como tamanho, cor, guia de néctar, volume e composição do néctar e quantidade de pólen são fatores importantes na atratividade das abelhas melíferas, podendo afetar a frequência de suas visitas (VARASSIN; AMARAL NETO, 2014). Estas diferenças interferem na frequência de visitação entre variedades e entre híbridos (KIILL et al., 2014). Assim, o objetivo desse trabalho foi registrar aspectos relevantes da floração incluindo a razão sexual e a descrição da morfologia floral de variedades de melancieira em cultivo orgânico.

\section{MATERIAL E MÉTODOS}

$\mathrm{O}$ experimento foi conduzido no período de janeiro à maio de 2014, na área agrícola do Centro de Agroecologia, Energias Renováveis e Desenvolvimento Sustentável (CAERDES), da Universidade do Estado da Bahia (UNEB), Campus III, Juazeiro-BA. A semeadura foi realizada em bandejas de poliestireno de 200 células, contendo substrato comercial, e após 14 dias foi realizado o transplantio, com espaçamento de 3,00 m entre fileiras e $0,50 \mathrm{~m}$ entre plantas. $\mathrm{O}$ delineamento utilizado foi o inteiramente casualizado, com quatro tratamentos (Congo, Elisa, Crimson Super e
Charleston Super) e quatro repetições e 50 plantas por parcela. O sistema de irrigação foi por gotejamento.

A adubação foi baseada na recomendação do Instituto Agronômico de Pernambuco (IPA) dividida em três etapas: etapa 1 - adubação de plantio (15 dias antes do transplantio), etapa 2 - adubação de cobertura (25 dias após o transplantio), etapa 3 - adubação de cobertura (40 dias após o transplantio).

$\mathrm{Na}$ adubação de fundação foram fornecidos esterco de aves $(600 \mathrm{~g} / \mathrm{planta})$, hiperfosfato de gafsa ${ }^{\circledR}$ (17 g/ planta), cinzas de madeira (50 g/planta), bórax ${ }^{\circledR}(5 \mathrm{~g} /$ planta), zinco (5 g/planta) e calcário dolomítico ( $200 \mathrm{~g} /$ planta $)$. Na $1^{\mathrm{a}}$ adubação de cobertura foram aplicados esterco de aves (450 g/planta) e cinzas de madeira (100 g/planta). Na $2^{\mathrm{a}}$ adubação de cobertura foi adicionado cinzas de madeira (300 g/planta).

A adubação foliar e o manejo fitossanitário foram realizados com a utilização de $0,25 \%$ Piroalho ${ }^{\circledR}, 0,05 \%$ Óleo de nim, $0,5 \%$ Óleo de algodão, $0,75 \%$ Piretron $^{\circledR}, 0,5 \%$ Litho cálcio $^{\circledR}, 0,5 \%$ Calda Sulfocálcica, $0,1 \%$ Bordasul $^{\circledR}, 0,1 \%$ Paracetic $^{\circledR}, 1 \%$ Sulfocal $^{\circledR}$ e $5 \%$ Biofertilizante líquido de acordo com as necessidades da cultura e a ocorrência de pragas e doenças.

Para o registro da floração, foram selecionadas ao acaso 20 plantas de cada variedade, sendo marcadas com fitas coloridas, e acompanhadas diariamente após a emissão das primeiras flores até o final da floração. Diariamente, foram anotadas em planilha o número de flores por planta e por tipo floral. Com estes dados foram obtidos o período de floração de cada variedade, bem como o período de produção de flores por tipo floral e a razão sexual.

Para os dados de morfologia floral, foram selecionadas ao acaso dez flores de cada tipo floral, por variedade, e realizadas as medidas do diâmetro da corola, cálice e ovário, e a altura da flor e do ovário. As medidas foram realizadas com paquímetro digital. Para as análises estatísticas foram utilizadas as médias e desvio padrão, com análise de variância e teste de Tukey utilizando-se o programa Statistica 6.0.

\section{RESULTADOS E DISCUSSÃO}

Para todas as variedades analisadas, foi registrada a emissão inicial de flores masculinas, permanecendo o seu número mais elevado que as femininas em todo o período de floração (Figura 1). Esta característica também foi registrada em plantas do meloeiro, o que parece ser um padrão característico em cucurbitáceas (SIQUEIRA, et al., 2011) Quanto às flores hermafroditas, a média registrada foi muito baixa em todas as variedades, com média de 1,45 flores por planta para a variedade Crimson a apenas 0,15 flores por planta na variedade Congo. 
Figura 1. Número médio de flores por tipo floral, em variedades de Citrulus lanatus, em cultivo orgânico, durante o período de floração, em Juazeiro-BA.
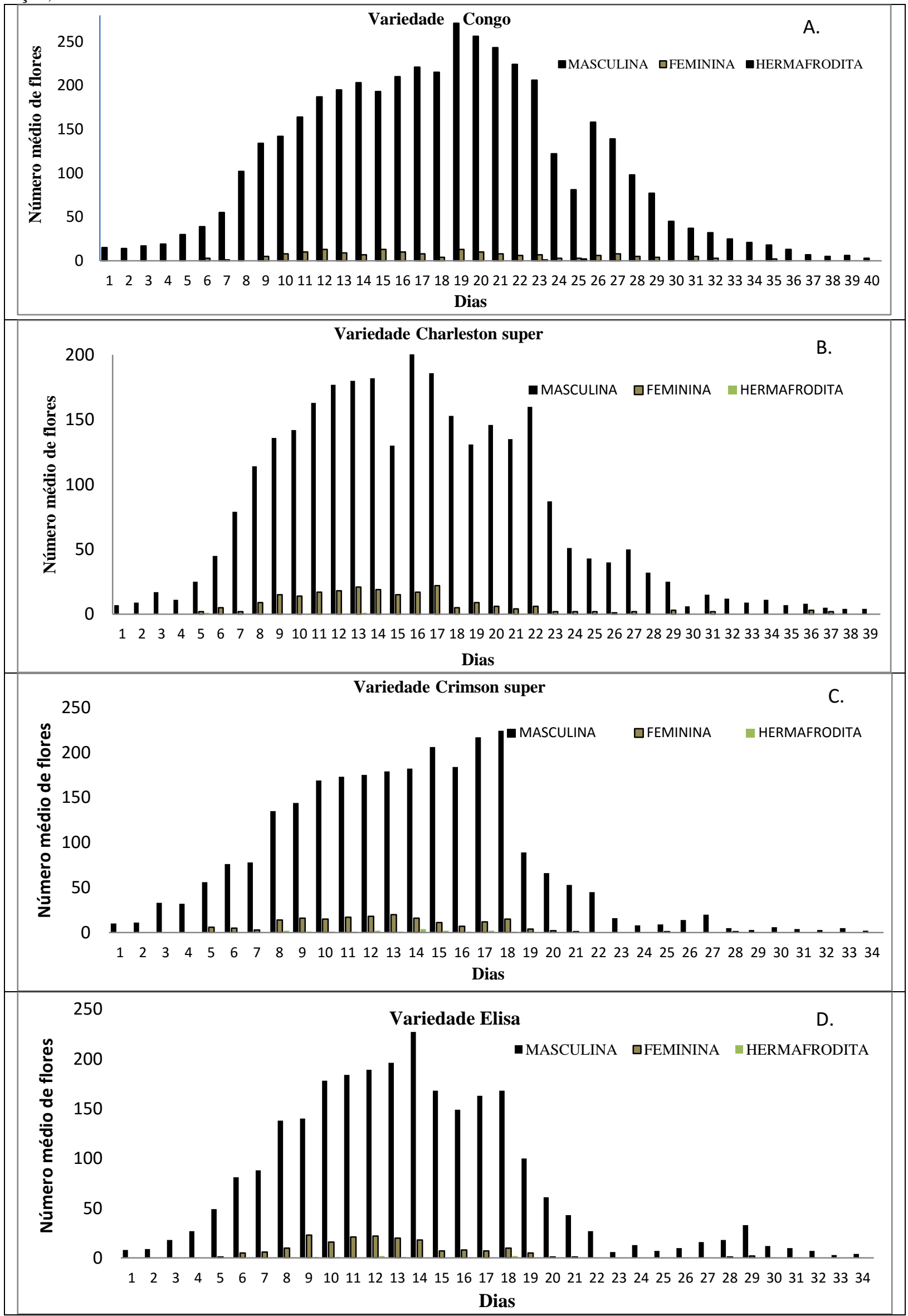
Quanto ao pico de floração, foi registrada variação entre as flores masculinas. Para a variedade Congo (Figura 1A), o pico foi registrado no $19^{\circ}$ dia estendendo-se até o $23^{\circ}$ dia. Por outro lado, a variedade Elisa (Figura 1D) foi a mais precoce apresentando o pico para as flores masculinas no $14^{\circ}$ dia. Para as outras duas variedades, esse período foi mais semelhante, entre o $15^{\circ}$ e o $22^{\circ}$ dia (Figuras 1B; 1C) Dessa forma, de acordo com a variedade podemos ter picos diferenciados, o que é relevante quando da introdução das colmeias no cultivo.

O pico de emissão de flores femininas foi registrado entre o $9^{\circ}$ dia e o $17^{\circ}$ dia de floração (Figuras 1A-D). Quanto a emissão da primeira flor feminina em relação a masculina, a variedade que teve a média mais alta foi a Congo com 9,05 \pm 3,28 e a menor foi a Charleston com 4,6 $\pm 2,81$ dias. Segundo Souza et al. (2008), na maioria das variedades de melancieira, as primeiras flores masculinas abrem-se cerca de três dias antes das primeiras flores femininas, diferindo assim dos dados aqui apresentados. Em polinização dirigida a emissão das primeiras flores femininas deve coincidir com a colocação das colmeias, caso ocorra atraso, pode haver perdas significativas na produtividade (BONFIM et al., 2013).

Não foi registrada diferença significativa entre o número médio de flores femininas por variedade. Quanto às flores masculinas, a maior média registrada foi para a variedade
Congo, seguida da Charleston (Tabela 1). A menor razão sexual foi registrada para a variedade Charleston em função da mesma apresentar a maior média de flores femininas por planta. Por outro lado, a variedade Congo foi a que apresentou a maior média de flores masculinas, obtendo assim a maior razão sexual.

Quando em plena floração, estudos apontam que a proporção de flores encontradas é de três a cinco flores masculinas para cada feminina (SOUZA et al., 2008). Dados semelhantes foram registrados no Estado de São Paulo com a variedade Crimson, em manejo convencional, apresentando razão sexual de 5,5 (SOUZA; MALERBO-SOUZA, 2005). Estes valores diferiram dos aqui registrados que apresentaram uma maior razão sexual para todas as variedades em estudo (Tabela 1).

Estudo realizado no Ceará, com híbridos do melão amarelo (Cucumis melo) revelou variações entre a razão sexual, com média de 6,1 a 11,3 (CRISÓSTOMO et al, 2004), o que pode ser atribuído a variabilidade genética. É importante ressaltar que o maior número de flores masculinas, além de servir como atrativo inicial para as abelhas polinizadoras, por ofertar pólen e néctar, são importantes na determinação do fluxo de pólen na população.

Tabela 01. Características da floração de variedades de melancieira em cultivo orgânico, em Juazeiro-BA.

\begin{tabular}{lllcll}
\hline & \multicolumn{3}{c}{ Flores } & \multicolumn{3}{c}{ Duração da floração } \\
\cline { 2 - 6 } Variedades & Femininas & Masculinas & Razão sexual & Masculina & Feminina \\
\hline Crimson & $8,25 \pm 2,22 \mathrm{a}$ & $120,95 \pm 19,13 \mathrm{a}$ & 14,66 & $28,10 \pm 4,25 \mathrm{a}$ & $11,65 \pm 4,42 \mathrm{a}$ \\
Charleston & $11,00 \pm 2,86 \mathrm{a}$ & $146,60 \pm 16,10 \mathrm{~b}$ & 13,32 & $31,55 \pm 4,65 \mathrm{~b}$ & $16,00 \pm 5,00 \mathrm{~b}$ \\
Congo & $8,70 \pm 2,65 \mathrm{a}$ & $209,30 \pm 2,82 \mathrm{c}$ & 24,05 & $35,25 \pm 2,31 \mathrm{c}$ & $20,00 \pm 2,85 \mathrm{c}$ \\
Elisa & $8,65 \pm 2,82 \mathrm{a}$ & $116,00 \pm 6,36 \mathrm{a}$ & 13,41 & $28,75 \pm 2,82 \mathrm{ab}$ & $11,90 \pm 2,12 \mathrm{a}$ \\
\hline p (Teste F) & 0,0058 & $<0,001$ & $<0,001$ & $<0,001$ \\
\hline \multicolumn{2}{l}{ Médias seguidas da mesma letra nas colunas não diferem entre si pelo teste de Tukey a 5\% de probabilidade }
\end{tabular}

As avaliações referentes às características morfológicas florais das variedades estudadas encontram-se nas tabelas 2 e 3. A análise de variância registrou diferença significativa entre as variedades e tipos florais para o diâmetro da corola $(\mathrm{F}=2,83 ; \mathrm{df}=7 ; \mathrm{p}=0,011)$ e altura da flor $(\mathrm{F}=10,89 ; \mathrm{df}=7$; p<0,001). Em ambos os tipos florais o diâmetro da corola pode variar entre 20 a $30 \mathrm{~mm}$ (WOLF, 1999). De acordo com as Tabelas 2 e 3, todas as variedades e tipos florais apresentaram média do diâmetro da corola dentro do referido intervalo.

Tabela 02. Características da morfologia floral de variedades de melancieiras avaliadas em flores femininas, em cultivo orgânico, em Juazeiro-BA.

\begin{tabular}{llllll}
\hline & \multicolumn{5}{c}{ Características florais } \\
\cline { 2 - 6 } Variedades & \multicolumn{1}{c}{$\begin{array}{c}\text { Diâmetro da } \\
\text { corola }(\mathrm{mm})\end{array}$} & Altura da flor $(\mathrm{mm})$ & $\begin{array}{c}\text { Diâmetro do } \\
\text { cálice }(\mathrm{mm})\end{array}$ & $\begin{array}{c}\text { Diâmetro do } \\
\text { ovário }(\mathrm{mm})\end{array}$ & $\begin{array}{c}\text { Altura do ovário } \\
(\mathrm{mm})\end{array}$ \\
\hline Congo & $20,87 \pm 3,94 \mathrm{a}$ & $38,79 \pm 7,22 \mathrm{a}$ & $3,03 \pm 1,13 \mathrm{a}$ & $6,27 \pm 1,84$ & $21,69 \pm 4,89$ \\
Charleston Super & $20,08 \pm 6,41 \mathrm{a}$ & $44,09 \pm 10,38 \mathrm{a}$ & $3,19 \pm 0,71 \mathrm{a}$ & $5,42 \pm 1,62$ & $18,92 \pm 4,09$ \\
Crimson Super & $20,42 \pm 3,88 \mathrm{a}$ & $22,37 \pm 2,83 \mathrm{~b}$ & $2,93 \pm 0,41 \mathrm{a}$ & $10,67 \pm 1,48$ & $15,98 \pm 2,76$ \\
Elisa & $24,65 \pm 4,10 \mathrm{a}$ & $33,83 \pm 6,91 \mathrm{ac}$ & $3,07 \pm 0,57$ a & $9,63 \pm 1,83$ & $13,16 \pm 1,76$ \\
\hline
\end{tabular}

Médias seguidas da mesma letra nas colunas não diferem entre si pelo Teste de Tukey a 5\% de probabilidade

Tabela 03. Características da morfologia floral de variedades de melancieiras avaliadas em flores masculinas, em cultivo orgânico, em Juazeiro-BA.

\begin{tabular}{lccc}
\hline & & \multicolumn{3}{c}{ Característica dos florais } \\
\cline { 2 - 4 } Variedades & Diâmetro da corola $(\mathrm{mm})$ & Altura da flor $(\mathrm{mm})$ & Diâmetro do cálice $(\mathrm{mm})$ \\
\hline Congo & $26,74 \pm 3,57 \mathrm{a}$ & $32,06 \pm 5,58 \mathrm{a}$ & $3,38 \pm 0,40 \mathrm{a}$ \\
Charleston Super & $23,61 \pm 5,27 \mathrm{a}$ & $37,32 \pm 12,34 \mathrm{ab}$ & $3,07 \pm 1,01 \mathrm{a}$ \\
Crimson Super & $22,30 \pm 3,52 \mathrm{a}$ & $27,70 \pm 5,06 \mathrm{a}$ & $2,79 \pm 0,43 \mathrm{a}$ \\
Elisa & $23,60 \pm 2,99 \mathrm{a}$ & $21,95 \pm 10,35 \mathrm{ac}$ & $2,63 \pm 0,28 \mathrm{a}$ \\
\hline
\end{tabular}

Médias seguidas da mesma letra nas colunas não diferem entre si pelo Teste de Tukey a 5\% de probabilidade 
Analisando as variedades entre si, com exceção da Elisa, todas as outras apresentaram flores masculinas com maior diâmetro da corola do que as femininas. Para a característica altura da flor, somente na variedade Crimson as flores masculinas apresentaram-se maiores que as femininas. $\mathrm{O}$ fato da maioria das flores masculinas apresentarem um maior diâmetro da corola, tornando estas flores mais visíveis, poderia justificar o maior número de visitas nas flores masculinas registrados por Araújo et al. (2014), em observações com esta mesma variedade. Além disso, esse tipo floral oferece néctar e pólen aos visitantes, o que as tornariam mais atrativas.

Em estudo realizado com híbridos do meloeiro (Cucumis melo) em Petrolina-PE, as flores hermafroditas apresentaram diferenças morfológicas em relação ao diâmetro da corola, sendo registrada também diferença com relação à altura em ambos os tipos florais, e entre os híbridos estudados. Segundo os autores, tais diferenças poderiam influenciar na atratividade e consequentemente na produtividade dessa cultura, uma vez que, a mesma é dependente dos serviços de polinização (KIILL et al., 2012)

Observando a tabela 2 , podemos perceber que as flores femininas da variedade Charleton e Congo apresentaram flores mais altas, enquanto que a variedade Crimson apresentou flores menores. Para as flores masculinas foi registrada diferença significativa apenas entre as variedades Charleston e Elisa (Tabela 3).

Outra característica importante na atratividade dos polinizadores é o diâmetro da corola, não sendo aqui registradas diferenças significativas entre as flores femininas, nem entre as masculinas, semelhante ao registrado para o diâmetro do cálice. Quando as medidas relativas ao ovário é importante ressaltar que, para cada variedade existe uma forma característica, assim não podemos fazer comparações entre os mesmos.

\section{CONCLUSÕES}

As variedades em estudo apresentaram diferenças na morfologia floral em relação ao diâmetro da corola e altura da flor, destacando-se a Elisa e Congo com maior corola e altura nas flores femininas e Congo e Charleston Super nas masculinas;

Variações na razão sexual foram registradas indicando a variedade Congo com maior emissão de flores, e a variedade Charleston Super com a menor;

O menor período de floração foi registrado nas variedades Crimson Super e Elisa.

\section{REFERENCIAS BIBLIOGRÁFICAS}

ALMEIDA, D. P. F. Cultura da Melancia. Faculdade de Ciências, Universidade do Porto, 2003, 9p.

ARAÚJO, D. C. Dos S.; SIQUEIRA, K. M. M. de; DUARTE, P. M. D.; SILVA, N. C. Comportamento de forrageamento de Apis mellifera na melancieira (Citrullus lanatus) no município de Juazeiro, BA. Revista Verde de Agroecologia e Desenvolvimento Sustentável, Pombal, v.09, nº 01, p. 59-67, 2014.

BONFIM, I. G. A.;CRUZ, D. O.; FREITAS, B. M.; ARAGÃO, F. A. S. Polinização em Melancia com e sem
Semente. Embrapa Agroindústria Tropical, 53 p. : il. ; (Documentos / Embrapa Agroindústria Tropical), 2013.

CRISÓSTOMO, J. R.; FALCÃO, L. F.;ARAGÃO, F. A. S.; FREITAS, J. G.; SILVA, J. F.; SANTOS F. H. C. JALMI, G. Biologia Floral do Meloeiro no Ceará: Emissão, Duração e Relação Flores Masculinas / Hermafroditas. Horticultura Brasileira, Brasília, v. 22, $\mathrm{n}^{\circ} 02$, Suplemento 02, 2004.

DENTON, O. A. Citrullus lanatus (Watermelon). In: Plant Resources of Tropical Africa. 2. Vegetables. Grubben, G.J.H. and Denton, O.A. (eds.). PROTA Foundation, Wageningen, Netherlands, 2004.

KIILL, L.H.P.; COELHO, M.S.; SIQUEIRA, K. M. M.; RIBEIRO, M. F.; COSTA, N. D.; FERNANDES, N. S.; SILVA, T. A. Relationship of floral morphology and biology of yellow melon hybrids with the attractiveness of pollinators. Magistra, Cruz das Almas, v. 24, número especial, p. 143-149, 2012.

KIILL, L.H.P.; SIQUEIRA, K. M. M.; COELHO, M. S.; SILVA,T. A.; GAMA,D. R. S.; ARAÚJO, D. C. S.; PEREIRA NETO, J. Frequency and foraging behavior of Apis mellifera in two melon hybrids in Juazeiro, state of Bahia, Brazil. Anais da Academia Brasileira de Ciência, Rio de Janeiro, v.04, nº86, p. 2050-2055, 2014.

NJOROGE G. N.; GEMMILL, B.; NEWTON, L. E.; NGUMI, V. W. Pollination ecology of Citrullus lanatus at Yatta, Kenya. International Journal of Tropical Insect Science, vol. 24, n. 1, p. 73-77, 2004.

SIQUEIRA, K. M. M.; KIILL, L. H. P.; GAMA, D. R. S.; ARAÚJO, D. C. S.; COELHO, M. S. Comparação do padrão de floração e de visitação do meloeiro do tipo amarelo em Juazeiro-BA. Revista Brasileira de Fruticultura, Volume Especial, E, p.473-478, 2011.

SOUZA, F. F.; DIAS, R. C. S.; QUEIRÓZ, M. A. Aspectos Botânicos. In: SOUZA, F. F. (Ed.). Cultivo da melancia em Rondônia. Porto Velho: Embrapa Rondônia, 2008, p. 11-15.

SOUZA, F. F.; MALERBO-SOUZA, D. T. Entomofauna visitante e produção de frutos em melancia (Citrullus lanatus Thunb.) - Cucurbitaceae. Acta Scientiarum, Maringá, v. 21, n. 3, p. 579-583, 2005.

VARASSIN, I. G. ; AMARAL NETO, L. P. . Atrativos. In: André Rodrigo Rech, Kayna Agostini, Paulo Eugênio Oliveira, Isabel Cristina Machado. (Org.). Biologia da Polinização. 1ed.Rio de Janeiro-RJ: Editora Projeto Cultural, 2014, p. 185-211.

WOLF, S.:LENSKY, : Y. PALDI, N. Genetic Variability in Flower Attractiveness to Honeybees (Apis mellifera L.) within the Genus Citrullus. Hortscience 34(5):860-863. 1999. 